\title{
Cortical and Subcortical Contributions to Stop Signal Response Inhibition: Role of the Subthalamic Nucleus
}

\author{
Adam R. Aron and Russell A. Poldrack \\ Department of Psychology and Brain Research Institute, University of California, Los Angeles, California 90095
}

\begin{abstract}
Suppressing an already initiated manual response depends critically on the right inferior frontal cortex (IFC), yet it is unclear how this inhibitory function is implemented in the motor system. It has been suggested that the subthalamic nucleus (STN), which is a part of the basal ganglia, may play a role because it is well placed to suppress the "direct" fronto-striatal pathway that is activated by response initiation. In two experiments, we investigated this hypothesis with functional magnetic resonance imaging and a Stop-signal task. Subjects responded to Go signals and attempted to inhibit the initiated response to occasional Stop signals. In experiment 1, Going significantly activated frontal, striatal, pallidal, and motor cortical regions, consistent with the direct pathway, whereas Stopping significantly activated right IFC and STN. In addition, Stopping-related activation was significantly greater for fast inhibitors than slow ones in both IFC and STN, and activity in these regions was correlated across subjects. In experiment 2, high-resolution functional and structural imaging confirmed the location of Stopping activation within the vicinity of the STN. We propose that the role of the STN is to suppress thalamocortical output, thereby blocking Go response execution. These results provide convergent data for a role for the STN in Stopsignal response inhibition. They also suggest that the speed of Go and Stop processes could relate to the relative activation of different neural pathways. Future research is required to establish whether Stop-signal inhibition could be implemented via a direct functional neuroanatomic projection between IFC and STN (a "hyperdirect" pathway).
\end{abstract}

Key words: striatum; frontal; activation; fMRI; cognitive control; imaging; Parkinson's disease

\section{Introduction}

Studies with the Stop-signal paradigm have shown that the right inferior frontal cortex (IFC) is critical for inhibiting an already initiated manual response (Aron et al., 2003a; Chambers et al., 2006). Such suppression could act at multiple foci in the motor system (De Jong et al., 1995; Band and Boxtel, 1999; van Boxtel et al., 2001; Aron et al., 2004). Here, we address the proposal that the target of frontal output is the subthalamic nucleus (STN), a subcortical region in the basal ganglia (Mink, 1996).

Recent work has specifically implicated the STN in Stopsignal response inhibition. STN stimulation improves Stopsignal reaction time (SSRT) in patients with Parkinson's disease (van den Wildenberg et al., 2006), and lesions to a midbrain region including STN slowed SSRT in rats (Eagle et al., 2004). As the STN sends excitatory output to the globus pallidus pars interna (GP) (Mink, 1996), STN activation could block the "direct" pathway fronto-striatal-pallidal discharges of the initiated response (Fig. 1A). Basal-ganglia models suggest STN activation could occur either via the "hyperdirect" fronto-subthalamic pathway or via the "indirect" fronto-striatal-pallidal-subthalamic pathway (Alexander et al., 1990; Mink, 1996; Nambu et al.,

Received Nov. 1, 2005; revised Jan. 11, 2006; accepted Jan. 19, 2006.

This work was supported by National Science Foundation Grant BCS-0223843. We thank Liat Levita, Dara Ghahremeni, and Roshan Cools for helpful comments.

Correspondence should be addressed to Dr. Adam R. Aron, Department of Psychology, Franz Hall, Box 951563, University of California, Los Angeles, CA 90095. E-mail: adamaron@ucla.edu.

DOI:10.1523/JNEUROSCI.4682-05.2006

Copyright $\odot 2006$ Society for Neuroscience $\quad$ 0270-6474/06/262424-10\$15.00/0
2002). For the Stop-signal paradigm, the hyperdirect pathway is prima facie a stronger candidate than the indirect one, because an already-initiated responses can be stopped in as little as $120 \mathrm{~ms}$.

We examined which regions would be involved in Going and Stopping using functional magnetic resonance imaging (fMRI) and the Stop-signal paradigm. Subjects performed choice responses but tried to inhibit their response when an occasional Stop signal occurred (Fig. $1 B$ ). Preoptimized sequences of Go trials, Stop trials, and null time (baseline) were used to maximize differences in fMRI signals between Stop and Go events. During scanning, the Stop-signal delay (SSD) was dynamically adjusted to yield a $50 \%$ successful inhibition rate so that SSRT could be estimated for each subject. This produced approximately equal proportions of StopInhibit trials (i.e., Stop trials without button press) and StopRespond trials (i.e., Stop trials with a button press). We predicted that: (1) Go-null would activate frontal, striatal, pallidal, and motor cortical regions, (2) StopInhibit-null would activate both Go regions and the right IFC and STN (for Stopping), and (3) StopInhibit-Go would activate the Stopping pathway more exclusively (by subtracting out regions related to the Go process). We also examined trials in which subjects could not stop (StopRespond) to see whether activation levels in regions related to Going and Stopping could account for failed inhibition. Region-of-interest (ROI) analyses were planned specifically for the IFC and STN to examine whether Stopping activation would predict SSRT and whether activation would be correlated among these regions of a putative stopping network. Finally, because the STN is a small structure and whole-brain 
A

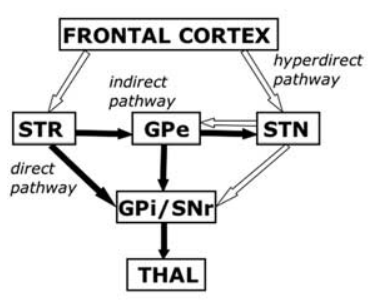

B

(otos

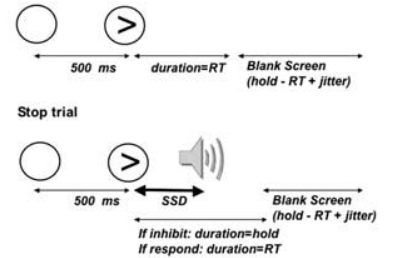

Figure 1. A basal-ganglia model and the Stop-signal paradigm. $A$, An influential model proposes three pathways through the basal ganglia (direct, indirect, and hyperdirect). SNr, Substantia nigra; THAL, thalamus; STR, striatum. Open arrows are excitatory (glutamatergic); filled arrows are inhibitory (GABAergic). Figure adapted from Nambu et al. (2002). B. The Stop-signal paradigm consists of Go- and Stop-signal trials. On Go trials, the subject has $1 \mathrm{~s}$ (the hold period) to make a left or right button press in response to the stimulus. As soon as the subject responds (reaction time), the stimulus is replaced by a blank screen for a variable period of time ( $1 \mathrm{~s}-\mathrm{RT}+$ jitter time, where jitter ranges between 0.5 and $4 \mathrm{~s}$, mean of $1 \mathrm{~s})$. $0 \mathrm{n}$ a Stop trial, a tone is played at some delay (SSD) after the arrow stimulus. If the response is inhibited, the arrow remains for $1 \mathrm{~s}$, followed by the blank screen jitter period; if the subject does not inhibit (i.e., responds), then the timing is the same as the Go trial. SSD changes dynamically throughout the experiment to produce $50 \%$ inhibition rate (see Materials and Methods).C,SSRT is estimated using the race model (Logan and Cowan, 1984). This assumes that $\mathrm{Go}$ and Stop processes are in a race and are independent of each other. The independence assumption implies that the distribution of Go processes on Stop trials (whether a response is made or not) is the same as the observed distribution of Go responses (when there is no Stop signal). On Stop trials, a tone occurs at some delay, the SSD after the Stop signal. If this delay is short, then $P$ (inhibit) is high and this is likely to be a Stoplnhibit trial; if the delay is long, then $P$ (inhibit) is low and this is likely to be a StopRespond trial. If SSD is varied so that $P$ (inhibit) $=0.5$, then SSRT can be estimated by subtracting the SSD from the median value of the Go distribution.

fMRI has limited spatial resolution, we ran a second experiment with additional subjects, using high-resolution functional and structural imaging protocols. Because the STN and immediate surrounding structures are visible as a hypo-intense midbrain region on T2-weighted scans (Dormont et al., 2004), localization of activation in this region could thus be confirmed.

\section{Materials and Methods}

Subjects. Thirteen right-handed healthy English-speaking subjects participated in experiment 1 (nine males; mean age, $29.2 \pm 4.5 \mathrm{SD}$ years), and five right-handed healthy English-speaking subjects participated in experiment 2 (one male; mean age, $23.8 \pm 3.7$ years). All subjects were free of neurological or psychiatric history and gave informed consent according to a University of California, Los Angeles Institutional Review Board protocol.

The Stop-signal task. The Stop-signal paradigm consists of a Go task and a Stop task (Fig. $1 B$ ). On each trial, a left- or right-pointing arrow stimulus was displayed on a computer screen. For the Go task, the subject responded as fast as possible with a left or right key press (using index and middle fingers of the right hand). For the Stop task (25\% of trials), the subject attempted to stop his/her response when a Stop signal was sounded at a particular SSD subsequent to the arrow stimulus. We used custom Matlab (MathWorks, Natick, MA) code to select sequences of Go, Stop, and null events and to select the distribution of null time in a way that optimized the detection of hemodynamic responses for the critical contrast of Stop and Go events. There were 32 Stop and 96 Go trials per scan (128 trials total). In every four trials, there was one Stop trial and three Go trials, and the number of leftward and rightward pointing arrows was equal. The SSD value for the Stop trial was sampled from one of the four staircases in turn (see below). Null events were imposed between every Stop or Go trial. The duration of null time ranged between 0.5 and $4 \mathrm{~s}$ (mean, $1 \mathrm{~s}$; sampled from an exponential distribution truncated at $4 \mathrm{~s}$ ). A large number of sequences was generated within these constraints, and the sequences with the highest efficiency to detect differences between Go and Stop events were selected (cf. Liu et al., 2001).

Performance on this paradigm has been characterized in terms of a race between Go and Stop processes (Logan and Cowan, 1984). The so-called "race model" assumes these processes run independently, and whichever finishes first determines whether the response is executed or inhibited. The duration of the Go process is directly observable through reaction time, whereas the duration of the stop process (known as the SSRT) must be estimated by observing the effects of varying the delay of the Stop signal (SSD). When the delay is short, the probability of inhibition is high; when it is long, the probability of inhibition is low (Fig. 1C).

A Go trial consisted of the following sequence. First, a white circular fixation ring (subtending $4.3 \times 6.2^{\circ}$ when viewed with scanner goggles) appeared in the center of the black background screen. After $500 \mathrm{~ms}$, a white arrow appeared within the fixation circle. The arrow pointed to the left on one-half the trials, and to the right on the other half. Arrow direction was randomized. The fixation ring and arrow remained on the screen for up to $1 \mathrm{~s}$ (limited hold), after which they disappeared and the background screen was shown for the null period. When the subject responded with a button press within the limited hold window, the fixation ring and arrow disappeared and the background was shown for the remainder of the limited hold plus the null period. If the subject pressed the wrong key in response to the arrow, the computer recorded it as an erroneous response. Such errors were extremely rare (see Results). A Stop trial was identical to a Go trial in all respects, except that a tone $(900 \mathrm{~Hz}$; duration, $500 \mathrm{~ms}$ ) was played at some SSD after the arrow stimulus. If the subject inhibited their response, then the arrow and fixation ring remained onscreen for the duration of the limited hold. If the subject responded, then the arrow and fixation ring disappeared, and the background screen was displayed for the remainder of the time. SSD changed dynamically throughout the experiment, depending on the subject's behavior. If the subject inhibited successfully on a Stop trial, then inhibition was made more difficult on a subsequent Stop trial by increasing the SSD by $50 \mathrm{~ms}$; if the subject did not successfully inhibit, then inhibition was made easier by decreasing the SSD by $50 \mathrm{~ms}$. Four step-up and step-down algorithms (staircases) were used in this way to ensure convergence to $P$ (inhibit) of $50 \%$ by the end of the experiment. The four staircases started with SSD values of 100, 150,200, and $250 \mathrm{~ms}$ respectively. In each block/scan of trials, there were 96 Go trials and 32 Stop trials. Each staircase therefore moved eight times within each block. The staircases were independent but randomly interleaved (i.e., each particular Stop trial belonged to one particular staircase, but the order of staircases was random trial-by-trial).

Procedure. For both experiments, subjects performed three blocks/ scans of the experiment ( 96 Go trials and 32 Stop trials per block). Directly before scanning, each subject was briefly trained to perform Go and Stop trials. It was made clear to subjects that Stopping and Going were equally important, and that it would not always be possible to stop. Within the scanner, subjects responded with their right hands on an MR-compatible button box. Stop tones were played through headphones at a level both sufficient to exceed scanner noise and comfortable to the subject. Each scan was preceded by an instruction screen with a reminder to the subject: "Remember, respond as FAST as you can once you see the arrow. However, if you hear a beep, your task is to STOP yourself from pressing. Stopping and Going are equally important." After each scan, subjects were given feedback in the form of median correct $\mathrm{RT}$ and number of discrimination errors on Go trials.

Behavioral analyses. As SSD was varied to yield $\sim 50 \% P$ (inhibit), SSRT was estimable by subtracting average SSD from median correct Go RT (according to the race model) This tracking procedure is ideal for producing a summary measure of SSRT, which is relatively robust against any violations of independence between Go and Stop processes (Band et al., 2003). Average SSD was computed, for each subject, from the values of the four staircases after the subject had converged on $50 \% P$ (inhibit). Values for the last 12 moves of each staircase were averaged to give a stable SSD estimate. This was highly reliable within subjects, as assessed by comparing the average of staircases 1 and 2 with staircases 3 and 4 
(experiment $1: n=13, r=0.93, p<0.0001$; experiment $2: n=5, r=0.94, p<0.05$ ). Additional measures of interest were number of discrimination errors on Go trials, median Go RT, and median StopRespond RT.

MRI data acquisition. Both experiments were run using a $3 \mathrm{~T}$ Siemens AG (Erlangen, Germany) Allegra MRI scanner. For experiment 1, we acquired 166 functional $\mathrm{T} 22^{*}$-weighted echoplanar images (EPIs) [slice thickness, 4 $\mathrm{mm} ; 33$ slices; repetition time (TR), 2 s; echo time (TE), $30 \mathrm{~ms}$; flip angle, $90^{\circ}$; matrix, $64 \times$ 64; field of view (FOV), 200]. In addition, a T2weighted matched-bandwidth high-resolution anatomical scan (same slice prescription as EPI) and magnetization-prepared rapid-acquisition gradient echo (MPRAGE) were acquired for each subject for registration purposes. The MPRAGE had the following parameters: TR, 2.3; TE, 2.1; FOV, 256; matrix, $192 \times 192$; saggital plane; slice thickness, $1 \mathrm{~mm}$; 160 slices. For experiment 2, we used a high-resolution EPI and T2-weighted turbo spin echo (TSE) protocol. We acquired 166 functional T2*-weighted EPIs (slice thickness, $3 \mathrm{~mm}$; 25 slices; TR, $2.2 \mathrm{~s}$; TE, $36 \mathrm{~ms}$; flip angle, 90; matrix, $128 \times 128$; field of view, 200), along with a matched-bandwidth high-resolution scan (same slice prescription as EPI). The TSE scan had the following parameters: TR, 3; TE, 14; FOV, 200; matrix, $256 \times 256$; slice thickness, $2 \mathrm{~mm}$; 160 slices. The orientation for matchedbandwidth, EPI, and TSE scans was coronal/oblique, with the slices chosen so as to give coverage from inferior prefrontal cortex to midbrain. TE for the EPI scan was chosen to maximize signal in the STN region by estimating $\mathrm{T} 2 *$ in this region using multiple echo times in a pilot subject. Stimulus presentation and timing of all stimuli and response events were achieved using Matlab and the Psychtoolbox (www.psychtoolbox.org) on an Apple PowerBook running Mac OS 9 (Apple Computers, Cupertino, CA).

Imaging preprocessing and registration. Initial analysis was performed using tools from the Centre for Functional Magnetic Resonance Imaging of the Brain software library (www.fmrib.ox.ac.uk/fsl). The first two volumes were discarded to allow for T1 equilibrium effects. The remaining images were then realigned to compensate for small head movements (Jenkinson et al., 2002). Translational movement parameters never exceeded 1 voxel in any direction for any subject or session. All images were denoised using MELODIC independent components analysis within FSL. Data were spatially smoothed using a $5 \mathrm{~mm}$ full-width-halfmaximum Gaussian kernel for experiment 1 and a $2 \mathrm{~mm}$ kernel for experiment 2. The data were filtered in the temporal domain using a nonlinear high-pass filter with a $66 \mathrm{~s}$ cutoff. For experiment 1 , a threestep registration procedure was used, whereby EPI images were first registered to the matched-bandwidth high-resolution scan, then to the MPRAGE structural image, and finally into standard [Montreal Neurological Institute (MNI)] space, using affine transformations (Jenkinson and Smith, 2001). For experiment 2, a two-step registration procedure was used, whereby EPI images were first registered to the matchedbandwidth high-resolution scan, then to the TSE image.

Model fitting. There were four variations of model fitting: (1) standard analysis for both experiments. The following events were modeled after convolution with a canonical hemodynamic response function: Go, StopInhibit, StopRespond, and nuisance events consisting of Go trials on which subjects did not respond or made errors. Events were modeled at the time of the arrow stimulus. Temporal derivatives were included as covariates of no interest to improve statistical sensitivity. Null events were not explicitly modeled and therefore constituted an implicit baseline. For each subject, and each scan, the following five contrast images were computed: Go-null, StopInhibit-null, StopRespond-null, StopInhibit-Go, StopInhibit-StopRespond. (2) Examination of arrow direction for experiment 1 . In this reanalysis, a similar model was fit, but Go trials were separately modeled as Go_left and Go_right according to arrow direction. (3) Examination of StopRespond-GoTrim. In this reanalysis, the Go distribution for each scanning run and subject was trimmed to match the StopRespond distribution for median RT (the removed Go observations were assigned to the nuisance variable). (4) Parametric analysis with SSD. This analysis examined the difference in Stopping activation when inhibition occurred early (i.e., soon after the Go process was initiated) compared with when it occurred late. The standard model was refit with the one added condition of mean normalized SSD as a regressor for StopInhibit trials. This analysis asks where in the brain StopInhibit-related activity increases with increasing SSD.

Statistical analysis (experiment 1). A higher-level analysis created across-session contrasts for each subject for a set of contrast images. These were then analyzed, at the whole-brain level, with random-effects analyses in SPM99, using one-sample $t$ tests. Group images were thresholded using cluster detection statistics, with a height threshold of $z>2.3$ and a cluster probability of $p<0.05$, corrected for whole-brain multiple comparisons [using Gaussian random field theory (GRFT)]. Further analyses used ROIs. The ROIs were originally defined as: (1) IFC, consisting of combined pars opercularis and pars triangularis from an automated anatomical labeling (AAL) atlas (Tzourio-Mazoyer et al., 2002) and (2) STN, consisting in a box of size $10 \times 10 \times 10 \mathrm{~mm}$, centered at MNI coordinates $10,-15,-5$, which provides full STN coverage according to an atlas of subcortical structures (Lucerna et al., 2002). In light of the strong results for pre-supplementary motor area (pre-SMA) and apparent GP (see below), we included two additional ROIs: (3) pre-SMA using the SMA region from the AAL atlas (with $y>0$ ) and (4) globus pallidus from the same atlas [this atlas does not separate globus pallidus pars interna (GPi) and globus pallidus pars externa (GPe)]. For ROI analyses, images were thresholded at $p<0.01$ uncorrected voxel level, and activation was regarded as significant if it survived $p<0.05$ for a cluster correction according to GRFT [and using local smoothness for small volume correction (SVC)].

Statistical analysis (experiment 2). Data were individually analyzed for each subject for the critical contrast of interest: StopInhibit-Go. A first analysis took the contrast and variance images from each run, merged them together, and produced a $z$-map for display purposes. This map was then transformed into a thresholded $t$-statistic activation map, which was then overlaid on the subject's TSE structural image. In a second analysis, MRIcro software (C. Rorden, University of South Carolina, Columbia, SC) was used to outline the midbrain hypointense area, which includes the STN (Dormont et al., 2004), on each subject's TSE scan, for left and right hemispheres separately. For each subject, $z$ values for each voxel (representing StopInhibit-Go) within the STN ROI were then averaged. A planned one-sample $t$ test $(\mathrm{df}=4)$ was run to assess whether the right STN was significantly activated for the group. A second contrast examined whether right STN activation was greater than left. A third contrast examined the Go-null contrast for left STN alone.

\section{Results}

\section{Behavior}

Median correct RT (on Go trials) and the estimate of SSRT for both experiments were in the typical range for young adults of similar age (Turner et al., 2002; Aron et al., 2003b), and the inhibition rate in both experiments was close to $50 \%$ (Table 1). Subjects made few discrimination errors on Go trials (fewer than three such errors on average). In neither experiment was Go RT significantly correlated with SSRT (experiment 1: $n=13, r=$ 
0.39 , NS; experiment 2: $n=5, r<0.1$, NS), consistent with the race-model assumption of independence of Go and Stop processes (Logan and Cowan, 1984). As expected by the race model (Logan and Cowan, 1984), median RT for Stop trials on which subjects responded (StopRespond trials) was significantly faster than median RT on Go trials (experiment 1: difference, $24.5 \mathrm{~ms}$, $t_{(12)}=6.7, p<0.0001$; experiment 2: difference, $13.2 \mathrm{~ms}, t_{(4)}=$ $1.5, \mathrm{NS})$.

\section{Experiment 1: whole-brain analyses}

The Go process activates fronto-striato-pallidal regions

Go-null significantly activated bilateral putamen, left thalamus/ STN/globus-pallidus, left motor cortex, and left SMA (Fig. 2A, supplemental Table 1, available at www.jneurosci.org as supplemental material). This Go network is consistent with many neuroimaging studies of motor responding, including previous studies of Go/No-Go (Liddle et al., 2001; Mostofsky et al., 2003) as well as with Mink's (1996) model of voluntary action, according to which responses occur via the fronto-striatal-pallidal pathway of the basal ganglia. There was also putative left STN activation for this contrast, which could relate to a postulated hyperdirect discharge before voluntary movement (to inhibit all potential responses) (Mink, 1996). However, because this was not replicated in experiment 2, we will not discuss this further. Although this Go network is mainly contralateral, consistent with a righthand response, we note that putamen activation was bilateral. Previous fMRI studies have found that whereas simple finger tapping movements activate controlateral putamen more than ipsilateral, more complex movement may activate bilateral putamen (Reichenbach et al., 1998; Mattay and Weinberger, 1999). The bilateral putamen activation was not an artifact of mixing leftward and rightward Go signals, because an auxiliary analysis found significant bilateral putamen activation for both Go_left versus null and Go_right versus null (see Materials and Methods, Model fitting).

The Stop process activates ipsilateral IFC, pre-SMA, STN, and GP StopInhibit-null significantly activated bilateral auditory cortex (the Stop tone was presented binaurally), bilateral thalamus/ STN/pallidum, medial frontal cortex, including pre-SMA and anterior cingulate cortex, bilateral putamen, parietal cortex, and bilateral orbital/insula cortex extending into the opercular IFC region in the right hemisphere (Fig. $2 B$ ). These activations reflect an already initiated Go process with a subsequent Stop process beginning, on average, $220.2 \mathrm{~ms}$ later (Table 1). To isolate the neural correlates specific to the Stop process, we directly contrasted StopInhibit-Go. There was significant activation of the right STN region, right IFC, right pre-SMA, and right GP, in addition to other regions such as right parietal cortex and right insula (Fig. 2C, supplemental Table 2, available at www. jneurosci.org as supplemental material). Although the pre-SMA region was not part of our hypothesis, we included it in our more detailed ROI analyses below because it was very robustly activated, because there is some indication in the literature of a role for medial frontal cortex in the inhibition of manual responses (Leimkuhler and Mesulam, 1985; Mostofsky et al., 2003), and because many neurophysiological studies in nonhuman primates have shown the importance of medial frontal cortex in oculomotor control (for review, see Schall et al., 2002).

For the moment, we note that these results are consistent with previous neuroimaging studies showing a right-lateralized "response inhibition network" including IFC, pre-SMA, right parietal cortex, and, sometimes, thalamic regions (Garavan et al., 1999, 2002; Liddle et al., 2001; Menon et al., 2001; Mostofsky et al., 2003; Rubia et al., 2003; Bellgrove et al., 2004). These results are also consistent with several event-related potential studies finding changes related to successful inhibition over right frontal (or even inferior frontal) regions (Pliszka et al., 2000; Schmajuk et al., 2005). We propose that the Stopping processes operates in the right hemisphere to excite the STN and suppress thalamocortical output. At some point, there is crossing over to affect left motor cortex. This is consistent with the fact that hemiballismus (disinhibited, flinging movements) are produced after lesions to the contralateral STN (for review, see Mink, 1996). Inhibitory effects on the left motor cortex are further revealed by considering differences between Go, StopInhibit, and StopRespond trials in left motor cortex (Fig. 2, top right peristimulus inset). Interestingly, StopRespond peaks at the same time as Go trials, but then there is a much stronger undershoot, as if the inhibition emerged too late and had later effects. In contrast, the StopInhibit trials show a peak activation that is delayed by $2 \mathrm{~s}$, as if the inhibition emerged in time to prevent activation from reaching a critical (response) threshold at a critical time. This finding provides blood oxygenation level-dependent (BOLD) evidence consistent with transcranial magnetic stimulation (TMS) studies showing that volitional response inhibition increases intracortical (GABAergic) inhibition in the motor cortex region (Sohn et al., 2002).

\section{StopRespond trials: when inhibition fails}

StopRespond trials activated a similar network to StopInhibit, including right IFC and STN, although inhibition was not successful (Fig. 2D). Combined with the observation (above) of the stronger undershoot of the BOLD response in left motor cortex for StopRespond compared with Go, this suggests the inhibitory process was triggered at the neural level, even if it was ineffective at the behavioral one. The reason for this must be because the Go process on these trials was too quick for inhibition to intercede: StopRespond trials were significantly faster than Go trials (see behavioral results) and, by definition of the race model, were faster than the Go process on StopInhibit trials. These findings are consistent with neurophysiological studies with an oculomotor version of the Stop signal paradigm, which found that presaccadic growth of activity in gaze-shifting neurons is correlated with saccade production, whereas the growth of activity in gaze holding neurons is correlated with saccade withholding (Hanes et al., 1998; Schall et al., 2002). The neural inhibitory mechanism may thus be triggered, but whether it is effective behaviorally may depend on whether the activity accumulates to some threshold. Examining when the inhibition becomes effective requires concurrent electromyography and measurement of force transduction for button presses, which we unfortunately did not do here. In the future, it would be interesting to relate such movement parameters to BOLD activation on so-called partial response trials (i.e., StopInhibit trials on which some EMG activity was discerned) to evaluate whether successful behavioral inhibition was the interruption of an already-initiated movement or rather of movement initiation.

To directly examine activation differences in Going- and Stopping-related regions, we contrasted StopInhibit and StopRespond. There was significantly more activation within bilateral putamen for StopInhibit than StopRespond (Fig. 2E, supplemental Table 3, available at www.jneurosci.org as supplemental material), but no significant differences at IFC, pre-SMA, GP, or STN. This finding of increased bilateral putamen activation for StopInhibit versus StopRespond has two potential explanations. It could signify a response inhibition process operating via the striatum (e.g., via the indirect fronto-striato-pallidal-STN path- 


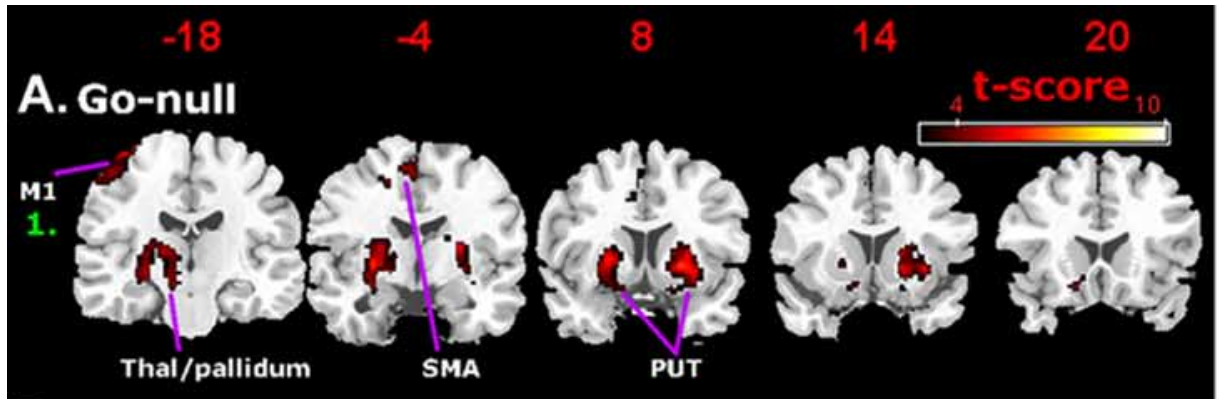

B. StopInhibit-null

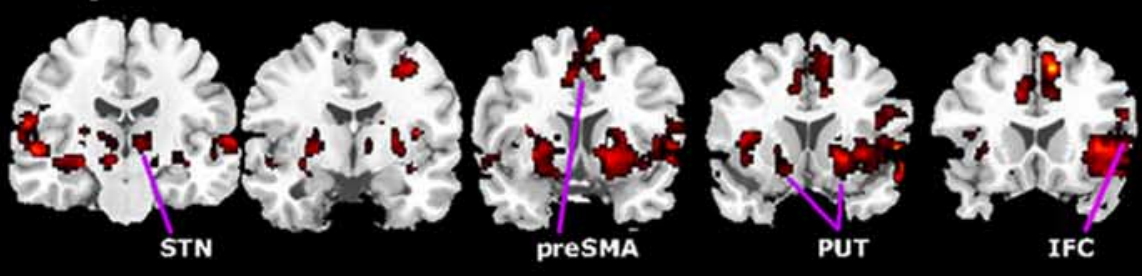

\section{StopInhibit-Go}



\section{StopRespond-null}



\section{E. StopInhibit-StopRespond}

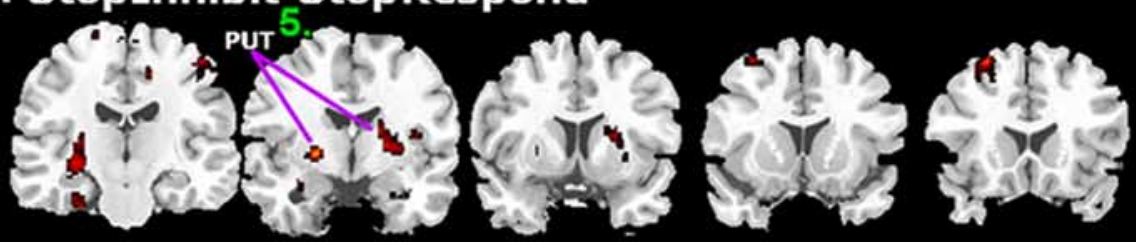

\section{F. StopRespond-GoTrim}

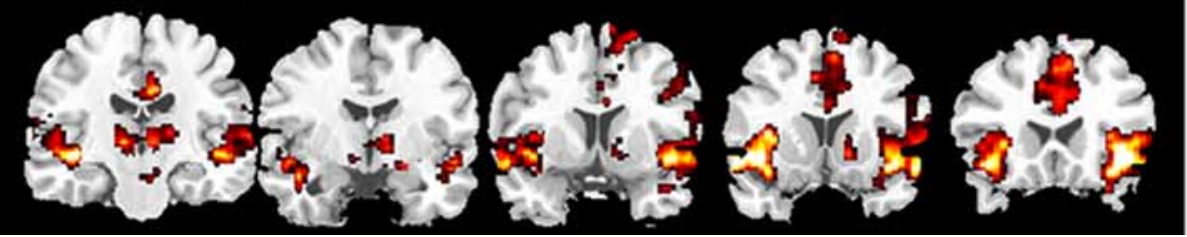

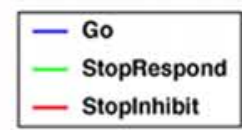

1. left M1 [42 -28 46]

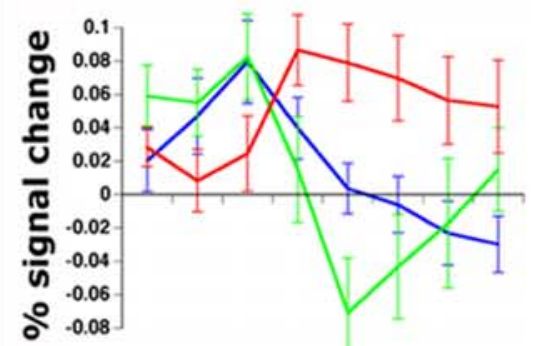

2. right STN [ 10 -14 -4]



3. right IFC [ $\left.\begin{array}{lll}44 & 12 & 8\end{array}\right]$



4. right pSMA [ $\left[\begin{array}{lll}6 & 20 & 50\end{array}\right]$

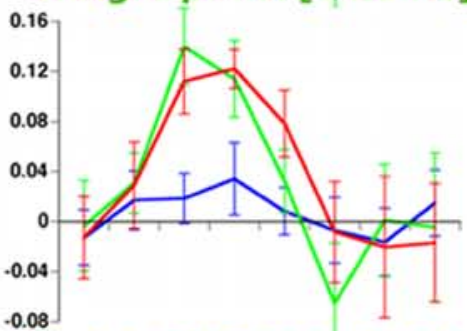

5. right PUT [ $\left.\begin{array}{llll}22 & -8 & -8\end{array}\right]$




way, which operates alone or together with a hyperdirect frontoSTN pathway), or it could signify increased BOLD activation associated with a slower Go process. We favor the latter explanation (of a slower Go process) for several reasons. First, the locus of increased activation of the putamen for StopInhibit versus StopRespond overlapped with a region activated by Go-null for which there was no response inhibition (Fig. 2 E, see inset panel 5 for estimates of hemodynamic response functions for these events). Second, a recent fMRI Stop-signal study (Vink et al., 2005) found that slower Go trials activated the dorsal striatum more than slower Go trials (within the context of a stop signal task). Third, neuropsychological evidence (reviewed in the Discussion) suggests that striatal damage may not critically affect SSRT.

Although increased putamen activation for StopInhibit trials is counterintuitive because StopRespond alone was associated with response execution, we note that BOLD activation of the putamen likely reflects the degree of response build-up in the fronto-striatal-pallidal pathway rather than being an index of response execution itself. We propose such response build-up is quicker for StopRespond trials and so activates putamen less. To support this interpretation, we performed a validation analysis in which we carefully matched response speed and contrasted StopRespond versus GoTrim trials (i.e., faster Go trials sampled from the Go distribution; see Materials and Methods, Model fitting). This contrast revealed a network almost identical to that for StopInhibit-Go (Fig. 2F). Notably, there was no significant activation of either left or right dorsal putamen, even at an uncorrected threshold of $p<0.05$ (effect size for StopInhibit-StopRespond was 2.1; effect size for StopRespond-GoTrim was -1.22 ; mean activation extracted from sphere of radius $3 \mathrm{~mm}$, centered in left putamen at MNI $-20,-10,2)$. This result shows that when speed of the Go process on Stop-signal trials (albeit unsuccessful ones) is matched for the speed of the Go process on Go trials, the putamen is not nearly so active as for the contrast StopInhibit-StopRespond. Therefore, putamen activation for the contrast of StopInhibit-StopRespond could relate to differences in the speed of the Go process. We suggest that future analyses examining striatal activation differences between conditions or groups of subjects should carefully control for response speed by using the StopRespond-GoTrim contrast.

\section{Experiment 1: ROI analyses}

The foregoing results show that fronto-striatal-pallidal regions were activated for the Go process, and IFC, pre-SMA, GP, and STN regions (especially dominant in the right hemisphere) were activated for the Stop process. If these Stopping regions together constitute a pathway, then their activation levels should significantly predict SSRT. Additionally, activation within these ROIs should be correlated and should be greater in the right than the left hemisphere. We tested these predictions by examining acti- vation within anatomically defined (see Materials and Methods) IFC, pre-SMA, GP, and STN ROIs. Unless indicated, statistical tests were two-tailed. We note that although other regions were clearly activated, such as the parietal cortex (previously implicated by many response inhibition studies), our hypothesis was to focus specifically on the targets of frontal inhibition within the motor system and to explore a putative fronto-subcortical circuit for response inhibition.

\section{The IFC/pre-SMA/GP/STN network is right-lateralized}

To specifically test whether response inhibition activation within the IFC, pre-SMA, GP, and STN was significantly greater in the right than left hemisphere, we extracted mean signal for each entire ROI for each subject in each hemisphere for the contrast of StopInhibit-Go (Fig. $3 A, B$ ). One-sample $t$ tests showed that the difference between StopInhibit and Go was significant for each of STN, IFC, GP, and pre-SMA in the right hemisphere (all $p<0.05$; Bonferroni's corrected), but not for the left hemisphere ROIs (all $p$ values NS). An ANOVA was performed with two hemispheres and three ROIs using these StopInhibit-Go difference scores. Overall activation was significantly greater in the right compared with the left hemisphere $\left(F_{(1,12)}=19.5 ; p<0.001\right)$, and this was also the case for each of STN, IFG, and pre-SMA (all $p<0.05$; post hoc tests), whereas GP showed a marginally significant effect when corrected for multiple comparisons. This analysis confirms for the entire, anatomically defined, regions of IFC, pre-SMA, GP, and STN that the activation for response inhibition is rightlateralized. This is unlikely an artifact of subjects using only their right hands in this experiment, because two previous fMRI studies found right IFC activation regardless of which hand was used (Konishi et al., 1998, 1999), and a study with TMS found that commission errors on Stop trials were increased after right IFC stimulation regardless of the hand to be inhibited (Chambers et al., 2006).

\section{IFC and STN activation is correlated}

If IFC, pre-SMA/SMA, GP, and STN regions together constitute a pathway, then their activation level could be correlated. Again, using mean StopInhibit-Go activation within the anatomically defined ROIs, we examined correlations between IFC and STN, STN and GP, and pre-SMA and STN in the right hemisphere. Across subjects, activation in STN was significantly correlated with IFC ( $n=13 ; r=0.68 ; p=0.011$, Bonferroni's corrected) (Fig. 3C), but the correlations between STN and GP, and STN and pre-SMA, were not significant.

IFC and STN activation is greater for subjects with faster SSRT Previously, we found that the degree of damage to right IFC in patients predicted SSRT (Aron et al., 2003a). Here, StopInhibit-Go activation within right IFC was indeed negatively correlated with

$\leftarrow$

Figure 2. Activated networks for Going and Stopping and key peristimulus time courses for the hemodynamic response. Images are in neurological format (right $=$ right). Activations are overlaid on coronal slices from the SPM single-subject structural template. All maps are thresholded at $t>3.05$ voxel level, $p<0.05$ cluster corrected for whole brain. $A$, Go trials activate contralateral motor cortex (M1), SMA, bilateral putamen (PUT), and left thalamus/pallidum, consistent with a fronto-striatal pathway. $\boldsymbol{B}$, Stoplnhibit trials activate auditory cortex, parietal cortex, right STN, medial frontal cortex, bilateral putamen, and bilateral orbital/insula cortex extending into the opercular IFC in the right hemisphere. These activations include those associated with preparing to execute a Go response as well as the response inhibition network revealed, more precisely, by the contrast of Stoplnhibit-Go (C). This network includes right-lateralized STN, IFC, GP, and pre-SMA (also shown on saggital and surface rendered images). This network is also activated on StopRespond trials (D).E, Stoplnhibit trials activate bilateral putamen more than StopRespond, but this is not the case for StopRespond-GoTrim $(\boldsymbol{F})$, which contrasts trials on which the subject tried to stop, but could not, with trials on the which the subject did not try, while controlling for response speed. On the right of the figure are shown peristimulus plots (with SEs) for different trial types for key foci. For these, mean activation for each subject was extracted from a sphere (radius, $4 \mathrm{~mm}$ ) centered on the peak group-activated voxel. Note the differential effects of Go, Stoplnhibit, and StopRespond on the time course of the estimated BOLD response in left motor cortex in particular (inset, top right): Stoplnhibit trials have a delayed response, whereas StopRespond trials peak at the same time and amplitude as Go trials but then show a strong undershoot as if the inhibition occurred too late. Surface rendering for the lateral view was created by mapping the group-averaged fMRI data into a population-averaged surface atlas using multifiducial mapping (Van Essen, 2005). Thal, Thalamus; acc, anterior cingulate cortex; ctx, cortex. 
SSRT, so that those subjects who inhibited more quickly activated this region more strongly. However, because the distribution of SSRT scores was more bimodal than continuous, we repeated this analysis as a between-samples $t$ test, using a median split to compare those seven subjects who inhibited fast (SSRT $<200 \mathrm{~ms}$ ) with those six subjects who inhibited slowly (SSRT $>200 \mathrm{~ms}$ ). Significant activation was again detected within a right IFC focus (MNI: 42, 26, $14 ; t=7.26$; $p=0.005$, SVC) and additionally within the right STN (MNI: $8,-20,-4 ; t=3.48 ; p=$ 0.051, SVC), but not for GP or pre-SMA (Fig. 3D,E). Therefore, those subjects who inhibited more quickly activated the right IFC and STN more, presumably to quickly block thalamocortical output. Similar analyses were performed for the contrast StopRespond-GoTrim. Subjects who inhibited more quickly activated the right STN significantly more (MNI: $14,-18,-4 ; t=3.97$; $p<0.05$, SVC), but there was no significant difference in the right IFC.

When inhibition occurs late versus early The above results show that right STN activation is correlated with IFC and is greater in subjects who inhibit more quickly. We examined whether STN activation would change as a function of how late the Stop process was initiated (i.e., by modeling StopInhibit trials with SSD as a parametric regressor). If the interval between Go and Stop is very small (or nonexistent, as in the case of a Go/No-Go test), it might be that response inhibition operates on an earlier different stage of the motor system (e.g., frontal planning regions). In contrast, when the interval is large, inhibition may operate at a later (more peripheral) stage (e.g., via the STN route), because the response is already near execution. Therefore, it was predicted that STN activation would be greater when the Go process was nearer completion than when it was in its initial stages. This prediction was borne out. The level of activation within right STN (MNI: 6, $-18,-2 ; t=$ 3.42 ; $p=0.028$, cluster corrected, onetailed) (Fig. $3 F$ ), as well as right pre-SMA (MNI: 6, 4, 68; $t=5.08 ; p<0.001$, cluster corrected) and right GP (MNI: 10, 2, 2; $t=$ 3.13; $p<0.05$, cluster corrected) but not right IFC, was positively correlated with SSD. Therefore, right IFC activation did not discriminate between early or late inhibition (it is apparently triggered regardless of the timing), but when the Go process was further underway, pre-SMA, GP, and STN were proportionately more active. This result suggests different mechanisms could govern response inhibition, depending on whether the response is still being planned or is already initiated. When the response is already close to execution (i.e., SSD is

B

D

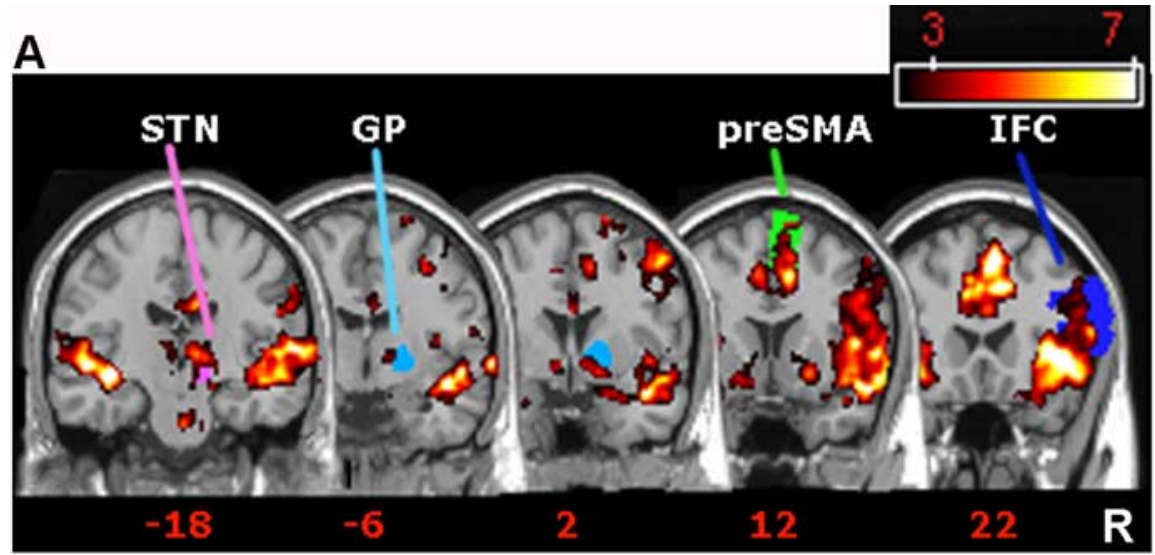

C
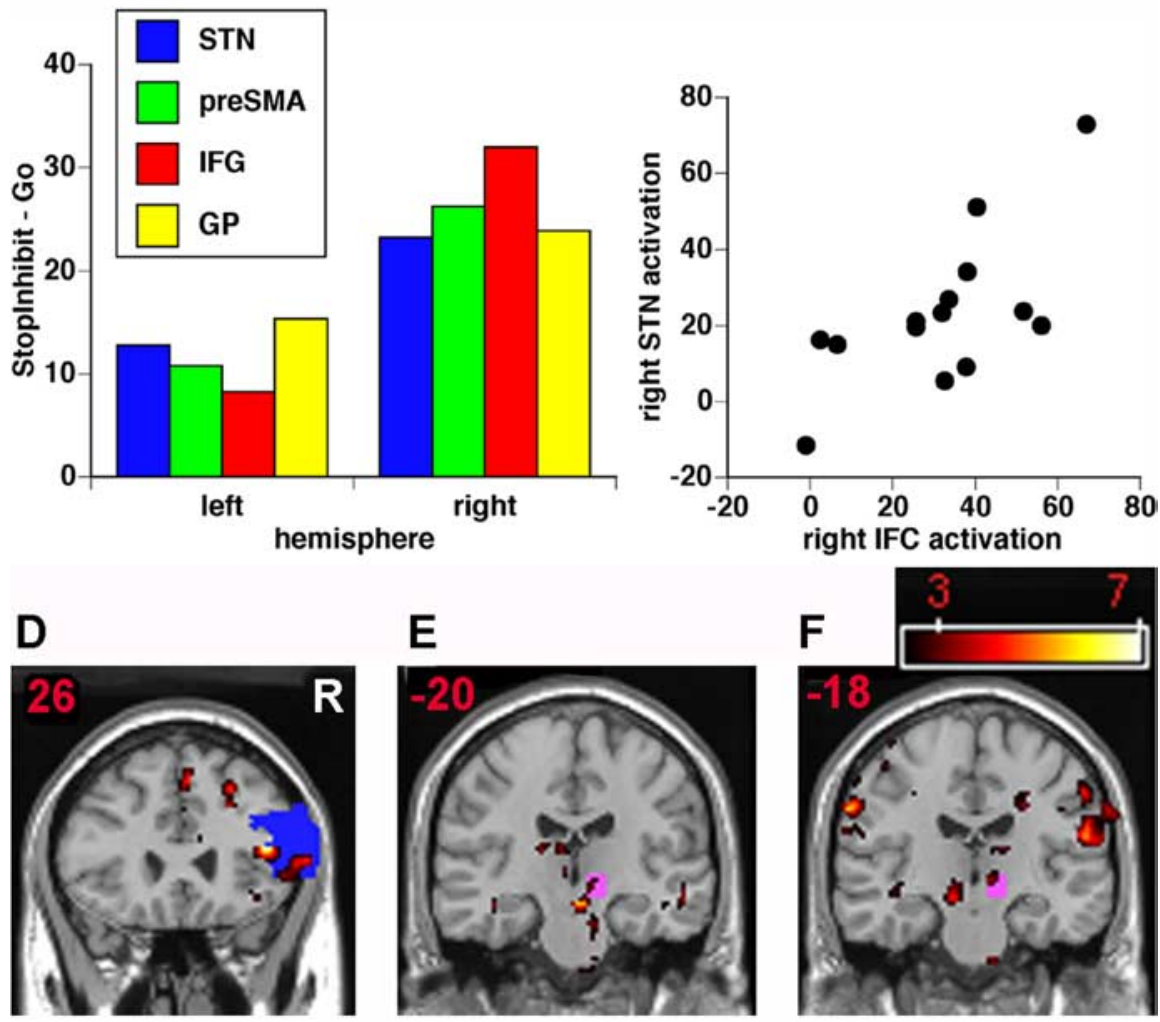

E

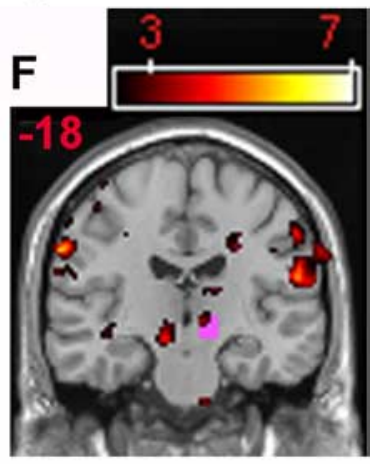

Figure 3. ROI analyses for experiment 1. B, Stoplnhibit-Go activation shown overlaid on anatomically defined IFC, pre-SMA, GP, and STN ROIs. The ROIs are color-coded. IFC pre-SMA and GP were defined from the AAL template (Tzourio-Mazoyer et al., 2002) and the STN according to the atlas by Lucerna et al. (2002). Activation is significant $p<0.05$ corrected for whole-brain multiple comparisons. $A$, The response inhibition network made up of IFC, pre-SMA, GP, and STN is significantly more active in the right than left hemisphere. For each subject, the mean value for all voxels in each anatomically defined ROI was computed for the contrast of Stoplnhibit-G0. All ROIs (except GP) showed significantly greater activation in the right hemisphere than the left (all $p<0.005$; Bonferroni's corrected). $C$, Across subjects, mean activation in right STN and right IFC was significantly correlated (for Stoplnhibit-Go contrast). D, E, Subjects who inhibit more quickly activate right IFC and STN more. Stoplnhibit-Go activation is significantly greater for subjects with fast SSRT versus those with slowSSRT (independentsamplesttest) in right IFC (MNI: $42,26,14 ; t=7.26 ; p=0.005$, SVC) and rightSTN (MNI: $8,-20,-4$; $t=3.48 ; p=0.051$, SVC). $\boldsymbol{F}$, When inhibition occurs later, STN is more active. For Stoplnhibit-Go, activation in right STN increases parametrically with increasing SSD (MNI: $6,-18,-2 ; t=3.42 ; p=0.028$, one-tailed, SVC). The $y$ coordinate in MNI space is shown above each coronal slice. The color scale represents tscore. $R$, Right.

relatively long), then there could be a greater role for a frontal/ basal-ganglia pathway targeted at the STN.

\section{Experiment 2: high-resolution imaging}

Because the STN is a small structure and whole-brain fMRI has limited spatial resolution, we ran a second experiment with new subjects, using high-resolution functional and structural (T2- 
A
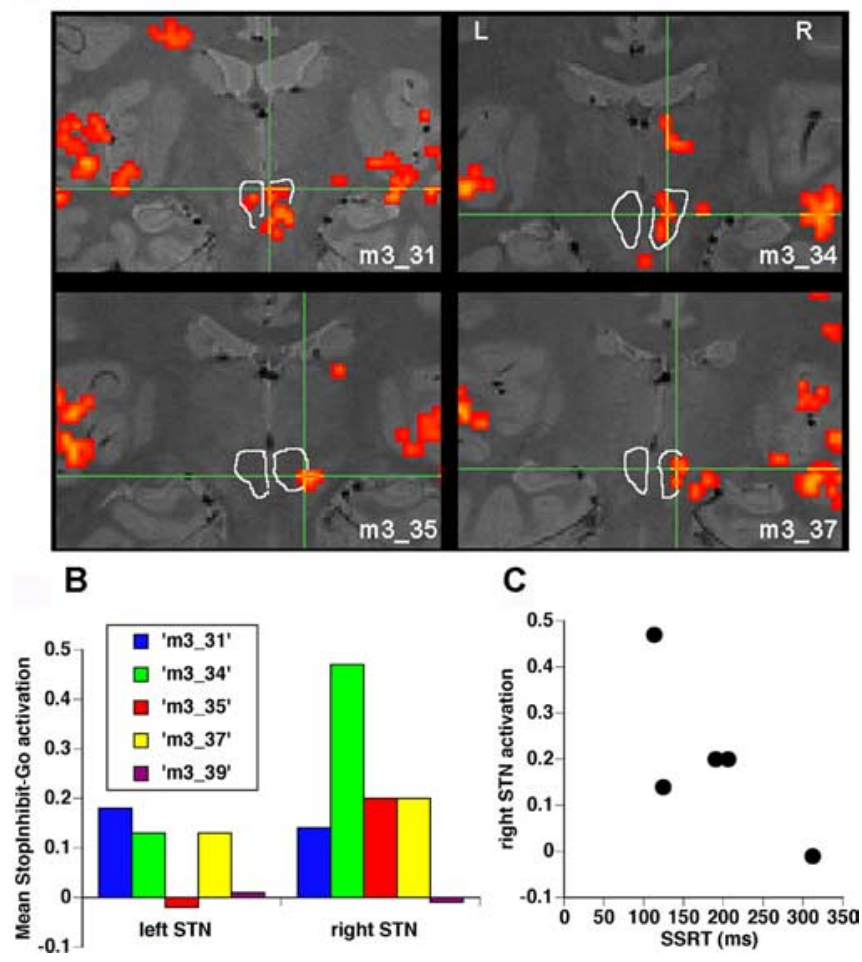

Figure 4. Subthalamic nucleus region is significantly activated by response inhibition in high-resolution experiment 2. A, Individual subject activation maps. Stoplnhibit-Go activation is shown for four subjects (the 5 th did not activate at this or any threshold). Activation is overlaid on each subject's T2-weighted structural scan. Images are in neurological format $(\mathrm{L}=\mathrm{L})$. For display purposes, individual subject maps were thresholded at $z>1.9$ and $p<0.05$ wholebrain cluster correction. Bilateral subthalamic nuclei are visible as hypointense (dark) regions in the midbrain (Dormont et al., 2004), demarcated by white lines. Activation of STN is evidently right-lateralized. There is also activation of bilateral auditory cortex in response to binaural Stop tone for all subjects. $\boldsymbol{B}$, Mean activation within STN in each hemisphere. $\boldsymbol{z}$-values for each subject, for the contrast Stoplnhibit-Go, were averaged within the entire hypointense STN region, for left and right hemispheres separately. As a group, the activation within right STN was significant $\left(t_{(4)}=2.65 ; p<0.05\right.$, one-tailed). There was a trend for right STN activation to be greater than left STN $\left(t_{(4)}=1.57 ; p=0.10\right.$, one-tailed). C, Activation within right STN correlated with SSRT: those subjects who inhibit more quickly activate more, but the small sample size precluded assessing this relationship statistically. L, Left; R, right.

weighted) imaging protocols. The STN was defined anatomically by manual tracing of a midbrain hypointense region on each subject's T2-weighted structural scan. Putative activation in the vicinity of STN in experiment 1 was confirmed in this experiment. Activation of the STN region was examined for contrasts of StopInhibit-Go and StopRespond-GoTrim. Activation for StopInhibit-Go was stronger and was present in four of five subjects at a threshold of $\mathrm{z}>1.9, p<0.05$, cluster corrected for the entire imaging volume (Fig. 4A). Activation was significant for the group as a whole in right STN for StopInhibit-Go $\left[t_{(4)}=2.65\right.$; $p=0.031$ (one-tailed)], and there was a trend for right STN activation to be significantly greater than left $\left[t_{(4)}=1.57 ; p=\right.$ 0.10 (one-tailed)] (Fig. 4B). The predicted correlation between right STN activation and SSRT was again observed: those subjects who inhibited more quickly had more activation (Fig. 4C).

\section{Discussion}

This study evaluated whether Stop signal response inhibition operates by activating the STN to suppress an initiated Go response. The results were consistent with this proposed mechanism. The Go process activated motor areas contralateral to the response hand including primary motor cortex, SMA, thalamus, and pallidum as well as putamen, consistent with the direct frontostriatal pathway, whereas the Stop process activated predominantly ipsilateral IFC, pre-SMA, GP, and STN. Furthermore, activation was significantly greater in IFC and STN for fast inhibitors than slow ones, was significantly correlated across subjects, and was significantly greater in the right than left hemisphere. A second high-resolution experiment verified significant stopping activation in the immediate vicinity of right STN. These results provide important new information about the functional neuroanatomy of cognitive control by verifying the importance of the STN for Stop-signal response inhibition and by suggesting that Go and Stop processes could relate to activation of different frontal/basal-ganglia regions.

An important qualification concerns the limitations of neuroimaging. Activations by themselves cannot prove that a particular brain region is sufficient (or even necessary) for a particular cognitive function (Poldrack, 2000; Rushworth et al., 2002). The interpretation of activations is also limited by the relatively poor spatial resolution of fMRI. Although we used high-resolution scanning in the second experiment, the hypointense region on the T2-weighted structural scans does include two nearby regions with high iron content (i.e., substantia nigra and the red nucleus) (Dormont et al., 2004). Therefore, we cannot be certain that our activations were specific to STN rather than to these other two regions. Although these inherent shortcomings limit our confidence that activations of IFC, pre-SMA, STN, and GP are specifically related to inhibition, we note that there exists strong convergent evidence from lesion, TMS, and deep brain stimulation studies implicating IFC and STN (at least) in Stop signal response inhibition.

We previously established the critical importance of the right IFC for Stop signal response inhibition (Aron et al., 2003a), and this was confirmed recently with TMS (Chambers et al., 2006). Other evidence implicates the STN: Parkinson's disease patients show abnormalities in STN firing (Garcia et al., 2005), have SSRT deficits (Gauggel et al., 2004), and benefit clinically from STN stimulation (Garcia et al., 2005). They also have significantly faster SSRT when their stimulators are on compared with off (van den Wildenberg et al., 2006). In rodents, lesions to a midbrain region including STN significantly slowed SSRT (Eagle et al., 2004).

Our fMRI results should be compared with neurophysiological studies using the Stop signal task in monkeys (Hanes et al., 1998; Schall et al., 2000) (for review, see Schall et al., 2002). Although these studies used an oculomotor countermanding task rather than one requiring manual responses, and although they only recorded within superior lateral and medial areas rather than inferior lateral ones, they demonstrate certain neurophysiological facts that likely transcend the difference in effectors. These studies show that the balance between gaze-shifting and gaze-holding neurons in such a region as the frontal eyefield (FEF) determines whether a saccade is produced. They also show that (1) whereas particular neurons within FEF enact inhibition before the SSRT, other neurons (visually responsive ones) do not, and (2) whereas FEF has neurons that control movement initiation, medial frontal regions do not but instead exhibit executive monitoring signals [for similar findings with fMRI, also see Curtis et al. (2005)]. These findings bear on our interpretation of IFC and pre-SMA activation in particular. It is indeed possible that these regions could be differentially activated by control and performance monitoring functions, respectively. Evaluating whether the medial frontal area plays a monitoring function requires performing a sequential-effects analysis to examine the 
relationship between activation and increased RT on Go trials after Stop trials. Unfortunately, we did not have sufficient power to perform that analysis here because our paradigm was optimized for detecting the difference between Go and Stop events. It is noteworthy that the critical importance of medial frontal regions for manual response inhibition has not, in any case, yet been established. One report did find that a single patient with a lesion of right SMA (but not one with a lesion of left SMA) had a deficit in response inhibition (Verfaellie and Heilman, 1987), but a re-examination of patients with medial frontal damage from our previous response inhibition study (Aron et al., 2003a) did not show that damage to this region critically affected SSRT (A. R. Aron, unpublished observations). In contrast, it is clearly established that the IFC is necessary for behavioral inhibition (Aron et al., 2003a; Chambers et al., 2006). Regarding the inability of summed BOLD activation to distinguish functionally heterogenous cells, it is indeed possible that the IFC activation reflects activity of cells that are movement-generating, others which are movement-holding, and still others which are unrelated to control. Functional MRI does not have the resolution to decide these questions, but future neurophysiological studies of this (and the STN) region could be helpful.

Although fMRI cannot, therefore, definitively reveal the specific functional role of subregions, our confidence that IFC and STN constitute an inhibitory network is increased by the fact that activation within these regions was correlated and also predicted SSRT, as well as by previous demonstrations of the importance of these regions for inhibition (reviewed above). Furthermore, we also note that tract-tracing studies in nonhuman primates show that ventral premotor cortex (a region highly similar to the pars opercularis region in humans with respect to its connectivity) (Croxson et al., 2005) projects directly to ipsilateral STN (for review, see Nambu et al., 2002), and we found coactivation of STN and IFC in the right hemisphere for response inhibition (Figs. 2, 3,4). Moreover, in the second, high-resolution experiment, activation of right STN was greater than left (Fig. 4). Further evidence that IFC and STN constitute a direct functional neuroanatomical pathway could be provided by diffusion tensor imaging of white-matter tracts in humans.

A question arises at to why previous response inhibition studies have not explicitly identified the STN (Garavan et al., 1999, 2002; Liddle et al., 2001; Menon et al., 2001; Mostofsky et al., 2003; Rubia et al., 2003; Bellgrove et al., 2004). This could relate either to insufficient resolution or because most used the Go/ No-Go paradigm. Because No-Go trials in the Go/No-Go paradigm are equivalent to stop trials with a zero SSD, the response is not yet initiated when the inhibit signal occurs. Hence, No-Go and Stop-signal response inhibition could be different processes regarding their point of contact with the motor system. Although No-Go activates right IFC just as Stopping does (for review, see Aron and Poldrack, 2005; Buchsbaum et al., 2005), and lesions of monkey homologues of IFC affect No-Go (Iversen and Mishkin, 1970; Petrides, 1986) just as lesions of right IFC affect SSRT in humans (Aron et al., 2003a; Chambers et al., 2006), No-Go frontal inhibition could suppress activity at an earlier point in the motor hierarchy (e.g., frontal motor planning regions) than Stop-signal inhibition does. Canceling an already initiated response given a Stop signal and canceling the development of a motor plan given a No-Go stimulus are clearly different processes. Consistent with this interpretation of different modes of inhibition, we found that STN activation on StopInhibit trials was greater the longer the SSD (i.e., when the Go process was closer to execution, successful inhibition engaged the STN more).
We tentatively suggest that inhibition in the stop signal paradigm (at least of the standard, stop-all-responses form) (cf. De Jong et al., 1995) is implemented via the hyperdirect pathway rather than the indirect one (which would require the striatum). First, SSRT has been observed at $<120 \mathrm{~ms}$ in some experiments, suggesting the appropriateness of a pathway with fewer synapses. Second, in two studies with early symptomatic Huntington's disease patients, we found that SSRT was not different from controls (Aron, 2003). Third, one study with a rodent model of the Stop signal paradigm found that ventral striatal damage did not affect SSRT (Eagle and Robbins, 2003a), whereas another (Eagle and Robbins, 2003b) found that medial striatal damage did affect estimates of SSRT but probably by altering the Go response more than affecting inhibition specifically. Such evidence suggests the striatum may not be critical for this form of inhibition, but additional research is clearly required.

In summary, we found activation of regions consistent with the direct fronto-striatal pathway for Going and of right IFC, STN, pallidum, and pre-SMA for Stopping. Although the role of pre-SMA in stopping manual responses remains to be clarified, the role of the IFC and STN seems clearer: these two regions could be nodes in either an indirect or hyperdirect frontal-subcortical pathway. We propose that right IFC excites STN, which excites GP and so suppresses basal-ganglia thalamocortical output, thus blocking the initiated Go response from being executed.

\section{References}

Alexander G, Crutcher M, DeLong M (1990) Basal ganglia-thalomocortical circuits: parallel substrates for motor, oculomotor, "prefrontal" and "limbic" functions. Prog Brain Res 85:119-146.

Aron AR (2003) The neural basis of inhibitory mechanisms in executive function. PhD thesis, University of Cambridge.

Aron AR, Poldrack RA (2005) The cognitive neuroscience of response inhibition: relevance for genetic research in attention-deficit/hyperactivity disorder. Biol Psychiatry 57:1285-1292.

Aron AR, Fletcher PC, Bullmore ET, Sahakian BJ, Robbins TW (2003a) Stop-signal inhibition disrupted by damage to right inferior frontal gyrus in humans. Nat Neurosci 6:115-116.

Aron AR, Dowson J, Sahakian BJ, Robbins TW (2003b) Methylphenidate improves response inhibition in adults with attention deficit/hyperactivity disorder. Biol Psychol 54:1465-1468.

Aron AR, Robbins TW, Poldrack RA (2004) Inhibition and the right inferior frontal cortex. Trends Cogn Sci 8:170-177.

Band G, Boxtel G (1999) Inhibitory motor control in stop paradigms: review and reinterpretation of neural mechanisms. Acta Psychol (Amst) 101:179-211.

Band GP, van der Molen MW, Logan GD (2003) Horse-race model simulations of the stop-signal procedure. Acta Psychol (Amst) 112:105-142.

Bellgrove MA, Hester R, Garavan H (2004) The functional neuroanatomical correlates of response variability: evidence from a response inhibition task. Neuropsychologia 42:1910-1916.

Buchsbaum BR, Greer S, Chang WL, Berman KF (2005) Meta-analysis of neuroimaging studies of the Wisconsin card-sorting task and component processes. Hum Brain Mapp 25:35-45.

Chambers CD, Bellgrove MA, Stokes MG, Henderson TR, Garavan H, Robertson IH, Morris AP, Mattingley JB (2006) Executive 'brake failure' following deactivation of human frontal lobe. J Cogn Neurosci, in press.

Croxson PL, Johansen-Berg H, Behrens TE, Robson MD, Pinsk MA, Gross CG, Richter W, Richter MC, Kastner S, Rushworth MF (2005) Quantitative investigation of connections of the prefrontal cortex in the human and macaque using probabilistic diffusion tractography. J Neurosci 25:8854-8866.

Curtis CE, Cole MW, Rao VY, D'Esposito M (2005) Canceling planned action: an FMRI study of countermanding saccades. Cereb Cortex 15:1281-1289.

De Jong R, Coles MG, Logan GD (1995) Strategies and mechanisms in nonselective and selective inhibitory motor control. J Exp Psychol 21:498-511.

Dormont D, Ricciardi KG, Tande D, Parain K, Menuel C, Galanaud D, Na- 
varro S, Cornu P, Agid Y, Yelnik J (2004) Is the subthalamic nucleus hypointense on T2-weighted images? A correlation study using MR imaging and stereotactic atlas data. Am J Neuroradiol 25:1516-1523.

Eagle DM, Robbins TW (2003a) Lesions of the medial prefrontal cortex or nucleus accumbens core do not impair inhibitory control in rats performing a stop-signal reaction time task. Behav Brain Res 146:131-144.

Eagle DM, Robbins TW (2003b) Inhibitory control in rats performing a stop-signal reaction-time task: effects of lesions of the medial striatum and D-amphetamine. Behav Neurosci 117:1302-1317.

Eagle DM, Baunez C, Shahl AP, Lehmann O, Robbins TW (2004) Inhibitory control in rats performing a stop-signal reaction time task: differential effects of lesions of the orbitofrontal cortex, infralimbic cortex and subthalamic nucleus. Soc Neurosci Abstr 30:781.12.

Garavan H, Ross TJ, Stein EA (1999) Right hemispheric dominance of inhibitory control: an event-related functional MRI study. Proc Natl Acad Sci USA 96:8301-8306.

Garavan H, Ross TJ, Murphy K, Roche RA, Stein EA (2002) Dissociable executive functions in the dynamic control of behavior: inhibition, error detection, and correction. NeuroImage 17:1820-1829.

Garcia L, D’Alessandro G, Bioulac B, Hammond C (2005) High-frequency stimulation in Parkinson's disease: more or less? Trends Neurosci 28:209-216.

Gauggel S, Rieger M, Feghoff TA (2004) Inhibition of ongoing responses in patients with Parkinson's disease. J Neurol Neurosurg Psychiatry 75:539-544.

Hanes DP, Patterson WF, Schall JD (1998) Role of frontal eye fields in countermanding saccades: visual, movement, and fixation activity. J Neurophysiol 79:817-834.

Iversen SD, Mishkin M (1970) Perseverative interference in monkeys following selective lesions of the inferior prefrontal convexivity. Exp Brain Res 11:376-386.

Jenkinson M, Smith S (2001) A global optimisation method for robust affine registration of brain images. Med Image Anal 5:143-156.

Jenkinson M, Bannister P, Brady M, Smith S (2002) Improved optimization for the robust and accurate linear registration and motion correction of brain images. NeuroImage 17:825-841.

Konishi S, Nakajima K, Uchida I, Kameyama M, Nakahara K, Sekihara K, Miyashita Y (1998) Transient activation of inferior prefrontal cortex during cognitive set shifting. Nat Neurosci 1:80-84.

Konishi S, Nakajima K, Uchida I, Kikyo H, Kameyama M, Miyashita Y (1999) Common inhibitory mechanism in human inferior prefrontal cortex revealed by event-related fMRI. Brain 122:981-991.

Leimkuhler ME, Mesulam MM (1985) Reversible go-no go deficits in a case of frontal lobe tumor. Ann Neurol 18:617-619.

Liddle PF, Kiehl KA, Smith AM (2001) Event-related fMRI study of response inhibition. Hum Brain Mapp 12:100-109.

Liu TT, Frank LR, Wong EC, Buxton RB (2001) Detection power, estimation efficiency, and predictability in event-related fMRI. NeuroImage 13:759-773.

Logan GD, Cowan WB (1984) On the ability to inhibit thought and action: a theory of an act of control. Psych Rev 91:295-327.

Lucerna S, Salpietro FM, Alafaci C, Tomasello F (2002) In vivo atlas of deep brain structures. Berlin: Springer.

Mattay VS, Weinberger DR (1999) Organization of the human motor system as studied by functional magnetic resonance imaging. Eur J Radiol 30:105-114.

Menon V, Adleman NE, White CD, Glover GH, Reiss AL (2001) Errorrelated brain activation during a Go/NoGo response inhibition task. Hum Brain Mapp 12:131-143.
Mink JW (1996) The basal ganglia: focused selection and inhibition of competing motor programs. Prog Neurobiol 50:381-425.

Mostofsky SH, Schafer JG, Abrams MT, Goldberg MC, Flower AA, Boyce A, Courtney SM, Calhoun VD, Kraut MA, Denckla MB, Pekar JJ (2003) fMRI evidence that the neural basis of response inhibition is taskdependent. Brain Res Cogn Brain Res 17:419-430.

Nambu A, Tokuno H, Takada M (2002) Functional significance of the cortico-subthalamo-pallidal "hyperdirect" pathway. Neurosci Res 43:111-117.

Petrides M (1986) The effect of periarcuate lesions in the monkey on the performance of symmetrically and asymmetrically reinforced visual and auditory go, no-go tasks. J Neurosci 6:2054-2063.

Pliszka SR, Liotti M, Woldorff MG (2000) Inhibitory control in children with attention-deficit/hyperactivity disorder: Event-related potentials identify the processing component and timing of an impaired rightfrontal response-inhibition mechanism. Biol Psychiatry 48:238-246.

Poldrack RA (2000) Imaging brain plasticity: conceptual and methodological issues-a theoretical review. NeuroImage 12:1-13.

Reichenbach JR, Feiwell R, Kuppusamy K, Bahn M, Haacke EM (1998) Functional magnetic resonance imaging of the basal ganglia and cerebellum using a simple motor paradigm. Magn Reson Imaging 16:281-287.

Rubia K, Smith AB, Brammer MJ, Taylor E (2003) Right inferior prefrontal cortex mediates response inhibition while mesial prefrontal cortex is responsible for error detection. NeuroImage 20:351-358.

Rushworth MFS, Hadland KA, Paus T, Sipila PK (2002) Role of the human medial frontal cortex in task switching: a combined fMRI and TMS study. J Neurophysiol 87:2577-2592.

Schall JD, Hanes DP, Taylor TL (2000) Neural control of behavior: countermanding eye movements. Psychol Res 63:299-307.

Schall JD, Stuphorn V, Brown JW (2002) Monitoring and control of action by the frontal lobes. Neuron 36:309-322.

Schmajuk M, Liotti M, Busse L, Woldorff MG (2005) Electrophysiological activity underlying inhibitory control processes in normal adults. Neuropsychologia 44:384-395.

Sohn YH, Wiltz K, Hallett M (2002) Effect of volitional inhibition on cortical inhibitory mechanisms. J Neurophysiol 88:333-338.

Turner DC, Robbins TW, Clark L, Aron AR, Dowson J, Sahakian BJ (2002) Cognitive enhancing effects of modafinil in healthy volunteers. Psychopharmacology (Berl) 165:260-269.

Tzourio-Mazoyer N, Landeau B, Papathanassiou D, Crivello F, Etard O, Delcroix N, Mazoyer B, Joliot M (2002) Automated anatomical labeling of activations in SPM using a macroscopic anatomical parcellation of the MNI MRI single-subject brain. NeuroImage 15:273-289.

van Boxtel GJ, van der Molen MW, Jennings JR, Brunia CH (2001) A psychophysiological analysis of inhibitory motor control in the stop-signal paradigm. Biol Psychol 58:229-262.

van den Wildenberg WPM, van Boxtel GJ, van der Molen MIW, Bosch DA, Speelman JD, Brunia CHM (2006) Stimulation of the subthalamic region facilitates the selection and inhibition of motor responses in Parkinson's disease. J Cogn Neurosci, in press.

Van Essen DC (2005) A Population-average, landmark- and surface-based (PALS) atlas of human cerebral cortex. NeuroImage 28:635-662.

Verfaellie M, Heilman KM (1987) Response preparation and response inhibition after lesions of the medial frontal lobe. Arch Neurol 44:1265-1271.

Vink M, Kahn RS, Raemaekers M, van den Heuvel M, Boersma M, Ramsey NF (2005) Function of striatum beyond inhibition and execution of motor responses. Hum Brain Mapp 25:336-344. 